\title{
Alltagssprachliche Mischungs- und Kreuzungsprozesse im urbanen Milieu: Zur sprachlichen Bricolage, Hybridität und Transgression
}

\section{Einleitende Bemerkungen}

In der vorliegenden Arbeit wird einer neuen, stark ethnisch geprägten Varietät des Deutschen, den Ethnolekt, Rechnung getragen. Gemeint ist die Mischvariante des Deutschen und zugleich eine Trendsprache unter Jugendlichen deutscher und nicht deutscher (insbesondere türkischer und arabischer) Herkunft, die sich in urbanen Ballungsräumen der Bundesrepublik etabliert hat und sprachliche Mischungen aus Elementen der Herkunftssprache ethnolektaler Sprecher und des Deutschen aufweist. Da die Betroffenen in der Bundesrepublik aufgewachsen sind, unterscheidet sich ihre Sprechart von der ihrer Vorfahren (der ehemaligen Gastarbeiter). Zudem weicht der ethnisch geprägte Sprechstil von der allgemeinen Jugendsprache ab, weil er nicht mehr als eine vorübergehende Sprachmode gilt, seine Aufmerksamkeit erregenden Merkmale an die deutsche Grammatik angepasst werden und die Sprecher selbst einem ähnlichen sozialen Milieu (den sogenannten „Ghettos") entstammen. Daher erscheinen die ethnolektalen Sprecher als diejenigen, die zwischen zwei unterschiedlichen Sprachen, Kulturen und Erziehungsstilen hin und hergerissen sind. Ihr Gemischtsprechen gilt dementsprechend als Ausdruck der soziokulturellen Identität, der Eigeninitiative; ihre Zugehörigkeit weder zur deutschen noch zur türkischen Sprache und Kultur manifest zu machen und sich stattdessen sprachlich (und kulturell) im „Zwischenraum“ zu positionieren.

Obwohl hier die Spezifika des ethnolektalen Sprechstils samt dem geschichtlichen Rahmen aufgegriffen und expliziert werden, wird das besagte sprachliche Phänomen nicht in den Vordergrund gerückt. Die vorliegende Arbeit hat es näm- 
lich zum Ziel, die zur Beschreibung der sprachlichen und kulturellen Hybridität dienenden Konzepte aufzuzeigen und zu beleuchten, in denen wie im Fall des Ethnolekts Sprache und Identität in unmittelbarer Beziehung und Wechselwirkung zueinander stehen. Aus diesem Blickwinkel betrachtet, verhelfen die Aufstellung und Aufhellung von Konzepten zu Sprachmischungs- und Kreuzungsprozessen dazu, jeweilige Diskurse über sprachliche Standardisierung und Nicht-Standardisierung theoretisch und sachbezogen zu untermauern.

Sofern die Konzepte zur Beschreibung der Sprachmischungs- und Kreuzungsprozesse von deren Initiatoren (Schöpfern) stammen, ist deren Ausgliederung, Zusammensetzung und Besprechungsart innovativ. Hierbei war die Vorgehensweise wie folgt: Zunächst wurde den deutschen und anerkannten linguistischen Arbeiten zum Phänomen Ethnolekt nachgegangen. Ein besonderer Fokus wurde dabei jeweils auf Strategien zur Bezeichnung der Mischungs- und Kreuzungsprozesse im deutschen Kultur- und Kommunikationskontext gelegt. Im Nachhinein wurde auf themenorientierte Ansätze (Theorien) eingegangen, die anderen Kultur- und Sprachkreisen entstammen und ihren deutschen Pendants als Matrize (ein wichtiger Bezugspunkt) gedient haben. Die Ergebnisse dieser Recherche wurden weiter systematisiert und in einer sinnvollen Reihenfolge zusammengebracht. Letztendlich wurden sie im Rahmen des 50. Linguistischen Kolloquiums in Innsbruck (3. - 5. September 2015) präsentiert, bisher aber nicht publiziert. Wie bereits eingangs erwähnt, sollte die vorliegende Arbeit den Diskursen zu sprachlichen Variationsmustern (dem sprachlichen Mischcode) im urbanen Milieu als theoretische Untermauerung dienen sowie über Sprachmischungs- und Kreuzungsprozesse Auskunft geben, an denen sich grundsätzlich Jugendliche deutscher und nicht deutscher Herkunft beteiligen und die längst in die Öffentlichkeit eingedrungen und Teil der medialen Realität sind.

\section{Auslöser für sprachliche Mischungs- und Kreuzungsprozesse}

Ausgangspunkt für Mischungs- und Kreuzungsprozesse im urbanen Milieu sind Migrationsverfahren und demographische Veränderungen, die die Etablierung von neuen kommunikativen Sozialstilen herbeigeführt haben. Das Erforschen des Sprachverhaltens der Hinzugezogenen sowie dessen Auswirkung auf den Sprachgebrauch der autochthonen Bevölkerung gilt als Domäne der interaktionellen Soziolinguistik oder Kontaktlinguistik. Hierbei liegt der Fokus der Mehrsprachigkeitsforschung grundsätzlich auf einer bestimmten Migrantengruppe: Sowohl in Europa als auch in Nordamerika gewinnen seit Ende der 1970er Jahre die Studien zu Arbeitsmigranten bzw. zu Gastarbeitern die Oberhand (vgl. Nelde 1998:518). Und dies ist ausgerechnet für die vorliegende Arbeit von hoher Relevanz. Denn 
es wird hier, wie bereits zuvor angekündigt, dem Sprachgebrauch Jugendlicher mit nicht deutschem Familienhintergrund, also der Nachkommen von ehemaligen deutschen Gastarbeitern nachgegangen, die zum großen Teil in der Bundesrepublik aufgewachsen sind. Hierbei weist ihre zur Interaktion mit der Mehrheitsgesellschaft verwendete Kontaktsprache zahlreiche und merkliche Abweichungen vom Standarddeutschen auf. Normferne Codes, die in Bildungskontexten, wo nach wie vor ein „monolingualer Habitus“ (vgl. Gogolin 1994) herrscht, geringgeschätzt werden, gelten längst auf Spielplätzen und Schulhöfen als ,ein selbstverständlicher Teil des unmittelbaren Lebenszusammenhangs, dem sich nur wenige Jugendliche entziehen können“ (Dirim/Auer 2003:58). In Anbetracht der zwei sprachlich orientierten Gebote, der Reinigung und Einsprachigkeit, deren Vergegenwärtigung und Anerkennung immer noch in deutschen Schulen gehuldigt wird, gilt Folgendes zu bedenken: „Das Modellieren der Sprache als homogenes Gebilde verstellt den Blick auf die sprachlichen Realitäten und die sie konstituierenden heterogenen kulturellen und sprachlichen Ressourcen“" (Erfurt 2003:25).

Die eingängige Annahme, das Migrationsverfahren habe die Grenzverschiebung und/oder Grenzüberschreitung von Sprachen und Sprachvarietäten bewirkt, ist nicht nur auf die heutige Sprachpraxis gemischtethnischer Gruppen zurückzuführen. Für unbestrittene Vorläufer der Sprachmischungsprozesse gelten nämlich Pidgins und Kreolsprachen, die sich aufgrund der Kontakte sowie Konflikte zwischen sprachlich dominanten und dominierten Gruppen herausgebildet haben, woraus auch deren sozialsymbolischer Charakter, sprich deren geringer sozialer Prestigewert hervorgeht. Die vorstehend genannten Kontaktsprachen sind für Kommunikationsanforderungen in den klassischen Kolonialländern charakteristisch. Sie fanden statt, wenn Sprecher anderer Erstsprachen aufeinandertrafen und miteinander agieren mussten. Als typische Kontexte galten hierbei Arbeitssituationen in Handel, Landwirtschaft und Produktion (vgl. Keim 2012:99). Aufgrund der Unkenntnis der Sprache der Aufnahmegesellschaft wurde ein stark reduziertes und auf den jeweiligen Arbeitskontext bezogenes Sprachkapital entwickelt, dessen Basis Wörter und grammatische Strukturen aus Herkunftssprachen jeweiliger Gesprächspartner stellten. Darauf weist Keim wie folgt hin: „In den klassischen Kolonialländern bildete die Sprache der sozial dominanten Gruppe, der Kolonialmacht, oft die Basis für das Pidgin, zumindest im Bereich des Wortschatzes. Im Bereich der Grammatik wurden die Strukturen aus den Sprachen übernommen, die für die beteiligten Sprecher einfach zu erlernen waren. Wenn z.B. eine Sprache ein komplexes morphologisches System hatte (Genus, Numerus, Kasus, Verbflexion etc.), die andere aber nicht, wurden die komplexen Formen vereinfacht oder weggelassen, z. B. Tempusformen auf Grundformen reduziert und Vor- und Nachzeitigkeit durch Zeitadverbien ausgedrückt" (Keim 2012:99). ${ }^{1}$

1 Siehe dazu noch Riehl (2009:120 - 121). 
Im Gegensatz zu Pidgin bezeichnet Kreol eine Erscheinung, wenn sich in über längere Zeiträume stabilen Kontaktsituationen pidginisierte Sprachvarietäten in voll ausgebaute Sprachsysteme umwandeln. Als Sonderform von Pidgin wird das Deutsch der ersten Gastarbeiter anerkannt, das dementsprechend als Gastarbeiterdeutsch (zum ersten Mal in einer Pilotstudie von Clyne 1968 beschrieben $^{2}$ ) oder Pidgindeutsch ${ }^{3}$ und im Falle der ersten Generation türkischer Gastarbeiter als Tarzanca ${ }^{4}$ bezeichnet wird. Im Hinblick auf die Erforschung des Gastarbeiterdeutsch ist eine Reihe von Projekten und Arbeiten entstanden. Erwähnt seien an dieser Stelle etwa das Heidelberger Forschungsprojekt „Pidgin-Deutsch“ (1975) oder die Veröffentlichungen von Bodemann/Ostrow (1975), Meisel (1975), Keim (1978), Yakut (1981), Dittmar (1982), Keim/Nikitopoulos/Repp (1982), Hinnenkamp (1982), Becker/Klein (1984) und Roche (1989). Da das Gastarbeiterdeutsch meist ungesteuert (ohne institutionelle Verankerung) erworben wurde und demnach stark pidginisierte und fossilisierte Formen des Deutschen aufweist, eignet es sich perfekt zur Markierung des ,asozialen Fremden“ und gleichzeitig auch zur Offenbarung der ihn beklemmenden Erfahrungen mit der Diskriminierung, Marginalisierung und Ausgrenzung (Ghettoisierung). Doch die Betroffenen erscheinen als „Akteure mit einer Sprachbiographie und dem kulturellen und ideologischen Gepäck ihrer Migrationsgeschichte auf, als Akteure, die die Sprachpraxis als ein Feld des sozialen Konflikts, der Grenzziehung und Marginalisierung erfahren und deren sprachliches

2 Gemeint ist eine Pilotstudie, an der sich insgesamt 15 Ausländer mit spanischer, griechischer, türkischer und slowenischer Erstsprache beteiligt haben. Aufgrund ihrer Aussagen war es für Clyne möglich, ein Pidginvarietäten-Gefüge festzulegen, das Einwortsätze, Ausfall von Verben, Fehlen des bestimmten und unbestimmten Artikels, Ausfall von Flexionsformen, Verwendung des Infinitivs, Generalisierung des femininen Artikels die, Verwendung von viel als Gradpartikel oder niks als Wort- und Satznegation auszeichnen.

3 Siehe hierzu (Riehl 2009:122 - 123): „Tatsächlich weist das Deutsch der Gastarbeiter (der 60er und 70er Jahre) Züge eines Pidgins auf. Das gilt vor allem für das Prinzip der Simplifizierung, das ein Grundmerkmal von Pidginsprachen ist. So fallen etwa im Gastarbeiterdeutsch verschiedene syntaktische Kategorien (Artikel, Personalpronomina, Präpositionen, Kopula) weg, Verbformen stehen im Infinitiv, Flexionsendungen fehlen und es besteht eine Tendenz zur Generalisierung des femininen Genus (vgl. Meisel 1975:21). Das Gastarbeiterdeutsch hat zudem mit Pidgins gemeinsam, dass es nur eingeschränkt gebraucht wird (nämlich am Arbeitsplatz) und auch als Verständigungssprache von Sprechern verschiedener nicht-deutscher Herkunftssprachen untereinander verwendet wird, z.B. von Gastarbeitern aus Italien, Griechenland und der Türkei.“

4 „Sowohl im Deutschen als auch im Türkischen weichen Muttersprachler in bestimmten Situationen und gegenüber solchen Sprechern, die sich durch die Eigenschaft ausweisen, daß sie der Muttersprache der ,ingroup' gar nicht oder nur beschränkt mächtig sind bzw. deren Gebrauch ihnen aus den unterschiedlichsten Gründen als ,defizitär' unterstellt wird, von den üblichen standardisierten und umgangssprachlich akzeptierten Sprachvarianten der Sprachgemeinschaft ab. Das sprachliche Produkt dieser spezifischen Abweichung können wir als simplifiziert bezeichnen. Unterschiedliche Sprachgemeinschaften haben dafür ihre eigenen Etikette geprägt, wobei ich in Anlehnung an die traditionelle linguistische Bezeichnung, diese Sprechweise als ,Foreigner Talk' bezeichnet habe. Aus Gründen der Unterscheidung habe ich für den türkischen FT den ethnographischen Terminus Tarzanca bzw. Tarzanisch gewählt“" (Hinnenkamp 1982:154, hervor. im Original). 
Repertoire die kulturellen Formen - und Brüche - ihres grenzüberschreitenden Lebens aufnimmt" (Erfurt 2003:25). Also haben die ersten Gastarbeiter aufgrund einer intensiven Auseinandersetzung mit den Ausgrenzungserfahrungen einen Sprechstil herausgebildet, den die bereits zuvor angeführten sprachlichen Besonderheiten kennzeichnen: Ausfall von Artikeln, Präpositionen, Pronomina, Kopulae; Ausfall bzw. Vereinfachung der Verbflexion (Numerus, Tempus, Modus, Person); übermäBige Verwendung des Infinitivs; Verbendstellung; Negationspartikel nix vor dem Verb; Übergeneralisierung mancher semantisch entleerter Verben (sein, haben, machen) oder Ausdruck der Vor- und Nachzeitigkeit durch Temporaladverbien.

Hierbei bilden Spezifika des Gastarbeiterdeutsch den Kern des sprachlichen Repertoires von nachkommenden Migrantengenerationen. Gemeint ist der Sprachgebrauch fremdstämmiger Heranwachsender in Deutschland, insbesondere türkischer Abstammung ${ }^{5}$, die inzwischen der dritten und vierten Migrantengeneration angehören. Der ethnolektale Sprechstil unmittelbar Betroffener, den türkische Interjektionen und Diskursmarker, Beschimpfungsformeln und Drohrituale, grammatische Reduktionen sowie phonetisch-prosodische Merkmale eines türkisch (ggf. ausländisch) klingenden Akzents ausmachen, ist längst der autochthonen jugendlichen Bevölkerung bekannt und von ihr als Ausdruck einer besonderen Solidarität mit sozial benachteiligten „Ghetto-Kids“ bzw. der von ihnen kreierten Subkultur (als ,Eintrittskarte' zu einer ghettoorientierten Jugendszene), zugleich aber auch - falls ironisch verfremdet und überproportional häufig zitiert - zur Distanzierung von derart „Ghettoisierten“ verwendet. Im engen Zusammenhang damit sei Folgendes angeführt: „Die markierte Verwendung stadtteilsprachlicher Elemente dient vor allem zur sozialsymbolisierenden Darstellung eines groben, aggressiven „Ghetto-Jugendlichen“, von dem sich die jungen Frauen distanzieren, zur Distanzierung von und zum aggressiven Spiel mit deutschen Lehrer/Betreuer und zur Herstellung von IngroupSolidarität gegenüber erwachsenen Deutschen“"(Kallmeyer/Keim 2003:44).

Hierbei werden zur Beschreibung des besagten Sprachphänomens verschiedene Termini und Definitionen gebraucht. Demnach ist der deutsch-türkische Mischcode sozial benachteiligter Jugendlicher unter den Namen wie etwa Kanak-Sprak (Zaimoğlu 1995, 1997), Türkendeutsch (Androutsopoulos 2001a:1, Kern/Selting 2006:239, Kern/Şimşek 2006:101-119, Şimşek 2012:155-180), Türkenslang (Auer 2003:255), Ghetto-/Ausländerdeutsch (Keim 2004:97, 2008:228), das (hybridolektale) Gemischtsprechen (Hinnenkamp 2000:96, 2007:195), Kiezdeutsch (Wiese 2009:782, 2012), Ethnolekt im Gegenwartsdeutsch (Lesch-Schumacher/ Schumacher 2009), Turbolekt (Wiese 2012:17) bekannt und verbreitet. Projekte, die die Mehrsprachigkeitserforschung in migrantisch geprägten Lebenswelten zum Ziel haben, schließen in methodischer Hinsicht an die Ethnographie der Kommunikation im urbanen Milieu, an die interaktionelle Soziolinguistik (Gumperz 1982, 1994)

5 „Das mehrsprachige Phänomen basiert im deutschen Migrationskontext vor allem auf deutschtürkischen Mischungen“(Canoğlu 2012:14, hervor. A. D.). 
sowie an die moderne Forschung zum Code-Switching (Rampton 1995, 1998) an. In Deutschland hat sich vor allem das Konzept des ,sozial kommunikativen Stils ${ }^{6}$ etabliert, das die Mannheimer Forschergruppe um Werner Kallmeyer, Inken Keim und Johannes Schwitalla erarbeitet hat (Kallmeyer (Hrsg.) 1994 u. 1995, Schwitalla 1995, Keim 1995, 2008 u. 2012). Dem Phänomen von alltagssprachlichen Kreuzungs- und Mischungsprozessen haben sich im Übrigen auch Jannis Androutsopoulos (2001a, 2001b, 2003), Peter Auer (2003), Inci Dirim und Peter Auer (2004) sowie Heike Wiese $(2006,2012)$ gestellt/verschrieben.

Insbesondere die Konzeption „des kommunikativen sozialen Stils“, die durch die Ethnographie der Kommunikation im Migrantenmilieu, den kultur-soziologischen Ansatz Bourdieus zur stilistischen Differenzierung in der hierarchisch geschichteten Gesellschaft (1979, deutsch 1982) sowie die Theorie der Aushandlung sozialer Ordnung in der Nachfolge des symbolischen Interaktionismus (Strauss 1968, Schütze 1987) geprägt ist, erlaubt es, die sozial-symbolische Bedeutung von normfernen Codewerten zu analysieren und zu erhellen. Demnach entscheidet das Gemischtsprechen über soziale Positionierung gemischtethnischer Gruppen und kann als deren wichtiger Identitätsausdruck wahrgenommen werden: „Die Ausprägung von Stil schafft Identitätssymbole, über die auch die Positionierung von Angehörigen eines Milieus bzw. von Akteuren einer sozialen Welt in einem übergreifenden gesellschaftlichen Rahmen möglich wird“" (Kallmeyer/Keim 2003:37). Sowohl beim Modell des „kommunikativen sozialen Stils“ als auch bei anderen Entwürfen zur Beschreibung der Mehrsprachigkeit gerät die symbolische Bedeutung der Interaktion in den Fokus. Gemeint ist der Input, der die Grenzüberschreitung und die damit einhergehenden Überlappung und Ineinandergreifen von eigenen und fremden Elementen, kurzum das hybride Ganze voraussetzt und determiniert. Dem wird jetzt bei der Charakterisierung von Projekten zur Mehrstimmigkeit des Gesprächs Rechnung getragen.

\section{Sprachmischungsprozesse und zu ihrer Beschreibung herangezogene Konzepte}

\subsection{Bricolage}

Ausgehend von Lévi-Strauss' Leistungen, entwickelt John Clarke das Konzept der Bricolage, das mit der „Neuordnung und Rekontextualisierung von Objekten gleichzusetzen ist, um neue Bedeutung zu kommunizieren“ (Clarke 1981:136). Demnach gilt Bricolage als eine besondere Art Stilbastelei, bei der dank der De- und Rekontextualisierung von den in der dominanten Kultur allgemein vorkommenden Strukturen die Integration alter und neuer Muster in ein homogenes Ganzes ermöglicht wird (vgl. Clarke 1981:137). Clarkes Konzept hat sich als prägend für die Jugendsprachforschung entpuppt. An das Motiv der Stilgestaltung/Stilkon- 
struktion, genauer gesagt an das der spielerischen Aneignung und Verfremdung von sprachlich-kulturellen Ressourcen anknüpfend, berichten Peter Schlobinski, Gaby Kohl, Irmgard Ludewigt von der Bricolage als einem „wesentlichen Kennzeichen jugendlicher Kommunikation" (Schlobinski/Kohl/Ludewigt 1993:112), Johannes Schwitalla $(1988,1994)$ von den „vielen Sprachen der Jugendlichen“, Peter Schlobinski und Niels-Christian Heins von „sprachlichen Versatzstücken aus unterschiedlichen kulturellen Hintergründen, [woraus] etwas Eigenes, Neues zusammengebastelt" wird (Schlobinski/Heins 1998:13). Zudem wird bei der letzteren Arbeit, „Jugendliche und ,ihre“ Sprache“, zwischen den Momenten der Selektion und Transformation unterschieden. In Anbetracht der sie voraussetzenden Verfahren wird die Transformation weiter in die mimetische und verfremdende Zitation ausdifferenziert. Während die mimetische Zitation als Schablone zur Identifikation fungiert (Es wird hier nämlich auf kulturelle Ressourcen zurückgegriffen, die der Lebenswelt der Beteiligten entstammen und mit denen sie sich identifizieren, vgl. Schlobinski/Kohl/Ludewigt 1993:49), wird bei der verfremdenden Zitation auf die Methode der Montage verwiesen, wo die emotionale Erschütterung aber auch Entlastung durch ironische Distanzierung und Verfremdung vollzogen wird. Im engen Zusammenhang damit bemerkt Markert, dass dabei Differenzschemata zu den eigenen kulturellen Ressourcen evaluieren (vgl. Markert 2009:11).

\subsection{Hybridität und sprachliche Hybridisierung im Migrationsmilieu}

Hierbei weist das Phänomen der Kreuzung und Mischung als Sonderform einer Praxis in mono- wie multilingualen Zusammenhängen Anknüpfungspunkte zu anthropologisch und kulturwissenschaftlich orientierten Erforschungen der Heterogenität im multikulturellen Raum und der Folgen des Kolonialismus und der Migration auf. Gestützt auf die in Lateinamerika geführte Postkolonialismus-Debatte mit Arbeiten von Néstor Garcia Canclini $(1989,1995)$ und Claudio Esteva-Fabregat $(1987,1995)$ wird an dieser Stelle auf das Konzept der Hybridität bzw. den Prozess der Hybridisierung eingegangen. Vorab sei angemerkt, dass der Begriff der Hybridität, der auf die Verfremdung des Reinen hindeutet und heutzutage neben Begriffen wie Kreolisierung und Synkretismus als ein viel gebrauchter Terminus zur Beschreibung von zunehmenden Heterogenisierungen in der modernen Welt gilt ${ }^{6}$, ursprünglich aus der Biologie stammt. In Naturwissenschaften steht das ,Hybride' für einen Mischling oder Bastard, „ein aus Kreuzungen hervorragendes Produkt von Vorfahren mit unterschiedlichen erblichen Merkmalen“ (Griem 2008:297). Sofern der besagte Begriff

${ }^{6}$ Vgl. hierzu Zapf (2002:40): „Der Begriff ,Hybridität‘ impliziert heute gerade die Weigerung, kulturelle Identität als ,rein' zu verstehen und wird oft für alles verwendet, was gemischter Herkunft oder Zusammensetzung ist". 
im 19. Jahrhundert vorwiegend zur Bezeichnung von einer (Tier-)Art oder Rasse gebraucht und abfällig bewertet wurde, referiert er im 20. Jahrhundert auf philologische und kulturtheoretische Phänomene, ohne dabei negative Kontexte hervorzurufen: „War er im Rassendiskurs des 19. Jahrhunderts dazu da, das negative Gegenbild des Bastards zu favorisieren, Reinheit zu diffamieren, so erfährt der Begriff Hybridität im 20. Jahrhundert eine Umdeutung, indem gerade im Vermischten Produktivität und Fruchtbarkeit gesehen wird. Außerdem dient er dazu, die Vorstellung, es gebe einen reinen Urzustand (der Sprache, der Kultur etc.) zu revidieren“"(Specht 2011:72).

So bedient sich Canclini des Begriffs der Hybridität zur Beschreibung von ökonomischen, kulturellen und demographischen Verhältnissen in den lateinamerikanischen Gesellschaften im späten 20. Jahrhundert, die Migration und Urbanisierung, zugespitzt formuliert: das Aufeinanderprallen von Elite- und Populärkultur, autochthoner Kultur und McDonaldisierung herbeigeführt haben. Im Endeffekt sind neue Formen kultureller Mischung und Durchdringung entstanden, die im krassen Gegensatzsowohl zu dem einen (eigenen) als auch dem anderen (fremden) Kultursystem stehen. So gesehen, bezeichnet Canclinis Konzept der Hybridisierung die Mischung und Verbindung von verschiedenen kulturellen Schemata, die durch die Interaktion gemischt-ethnischer Gruppen hergestellt wurden. Hierbei betrachtet er das Mischungs-Verfahren als eine Verschiebung von Grenzen, die „eine Erosion alter Identifikationsmuster" (Rincón 1994:28) bewirkt hat.

Wie bereits erwähnt, findet der Terminus Hybridität auch im sprachwissenschaftlichen Bereich Verwendung. Der Sprachwissenschaftler Volker Hinnenkamp bezeichnet als Hybrid bzw. Gemischtsprechen den Sprachgebrauch Jugendlicher deutscher und nicht deutscher Herkunft in migrationsbedingten bundesdeutschen Kontexten. Hierbei wird wie folgt argumentiert: „Hybrid lässt sich laut Duden umschreiben als ,aus Verschiedenartigem zusammengesetzt, von zweierlei Herkunft, gemischt, zwitterhaft“, das Verb hybridisieren wird schlicht mit „bastardisieren" gleichgesetzt (Duden. Deutsches Universalwörterbuch 2001:810). Wer mischt, zusammensetzt, bastardisiert etc., widerspricht, ja widersetzt sich dem wie immer fiktiven Reinheitsgebot. Nicht ganz zufällig verbergen sich dahinter nicht unbedingt Schwäche, Hilflosigkeit und Flickschusterei, sondern implizite wie explizite Selbstverständnisse; Identitäten, deren Träger und Trägerinnen diesen Schritt, Sprachgrenzen zu überspringen, als Antwort und Reaktion auf bestimmte sprachliche, sprachpolitische und historische Umstände, aber auch herausgefordert durch sie unternehmen“ (Hinnenkamp 2005:7).

\subsection{Language crossing}

In der Sozio- und Kontaktlinguistik werden hybride Praktiken als language cros$\operatorname{sig}^{7}$ aufgefasst. Sie kommen dann zum Vorschein, wenn „Sprecherinnen und

\footnotetext{
7 Von Hinnenkamp (1998) wird als deutsches Äquivalent sprachliche Kreuzung bzw. Sprachkreuzung gewählt.
} 
Sprecher in ihnen,fremde ${ }^{6}$ Territorien hineinwandern. Im Gegensatz zu klassischen Konstellationen von Zwei- und Mehrsprachigkeit, wie sie zum Beispiel bei bilingualen Familien und Migrantengemeinschaften vorliegen, geht es beim Crossing um unorthodoxe Fälle von Sprachkontakt, um fragmentarische, unnormierte und unerwartbare zwei- oder mehrsprachige Praxis“ (Androutsopoulos 2003:79, hervor. im Original).

Erste Berichte zum Crossing-Verfahren entstammen englischen Sprachinstituten und sind grundsätzlich mit den Veröffentlichungen von Roger Hewitt (1986) und Ben Rampton $(1995,1998)$ dokumentiert. In den Arbeiten von Hewitt wird zwischen einer ethnozentristischen kulturellen und einer suprakulturellen Modalität unterschieden. Während das erste Konzept eine hybride bzw. synkretistische Kultur bezeichnet, die der Ethnizität als Gesamtprinzip huldigt, aber dennoch auf einheitlichen Wertvorstellungen basiert, wird mit dem zweiten Konzept auf eine außerethnische, von lokalen Besonderheiten emanzipierte Kommunikation verwiesen (vgl. Hewitt 1998:19). Zur Beschreibung einer komplexen Sprachkontaktkonstellation in einer südenglischen Stadt, wo drei Sprachen, und zwar afro-karibisches Kreol, Panjabi (eine neuindische Sprache) und stilisiertes AssianEnglisch zur Interaktion eingesetzt wurden, griff Ben Rampton auf John. J. Gumperz' Konzept des metaphorischen Code-Switchings zurück. Es sei vorab betont, dass sich Gumperz der allgemeingültigen Auffassung vehement widersetzte, als sei der Sprachwechsel Ausdruck sprachlicher Inkompetenz und zugleich Beweis für jegliche Ausweichmanöver: „Anstatt von einem sprachlichen Defizit auszugehen, wird Code-Switching nach Gumperz als Element sozialer und rhetorischer Bedeutung verstanden, das von Sprechern gebraucht wird, um ihre Adressaten auf den jeweiligen sozialen und situativen Kontext des Gesprächs aufmerksam zu machen" (Bücker 2007:14). Gumperz differenziert zwischen einem situativen und metaphorischen Code-Switching. Bei den besagten Ausprägungen von Code-Switching ist das Element des (Un)erwarteten bzw. die Erwartungshaltung maßgebend. Aufgrund dessen wird der situative Sprachwechsel in multilingualen Kontexten routiniert eingesetzt, als konventionalisiert und denkbar angesehen. Er findet dann statt, wenn eine Situation und herrschende Normen einer Interpretation einer anderen (kulturellen) Perspektive bedürfen, was nicht nur eine Änderung nicht nur bei Gesprächsthemen und -strategien, sondern auch bei Verpflichtungen für die Gesprächsteilnehmer impliziert (vgl. Blom/Gumperz 1972:409). Obwohl die betroffenen Interaktanten von einer Sprachperspektive in die andere wechseln, werden jegliche dabei auftretenden Differenzierungen auf lexikalischer und nicht morphologisch-syntaktischer Ebene abgezeichnet (vgl. Blom/Gumperz 1972:431). Während das situative Code-Switching situationsbedingte Faktoren wie Gesprächspartner, -thema oder -ort voraussetzt, kommt das metaphorische Code-Switching dann vor, wenn eine Stellungnahme zu dem bereits Gesagten abgegeben werden soll, was gewöhnlich auf eine veränderte Absicht, Einstellung oder Gefühlslage beim Sprecher hindeutet (vgl. Bücker 2007:15). Wie bereits erwähnt, definiert Rampton Language Crossing als Form des von Gumperz be- 
schriebenen metaphorischen Code-Switchings. Dass der Sprachwechsel nicht in Routine-, sondern in Grenzsituationen erfolgt, bringt die besagten Konzepte (das metaphorische Code-Switching und Language Crossing) auf einen gemeinsamen Nenner. Die Tatsache, dass sich am Sprachwechsel Personen aus einer anderen Sprachgemeinschaft beteiligen (im Originaltext ist Crossing „the use of language varieties with social or ethnic groups that the speaker does not normally belong to" (Rampton 1995:14) bzw. „the use of a language not normally felt to belong to the speaker" (Rampton 1998:291)), macht die Überschreitung der ethnischsozialen Grenze evident. Dies findet allerdings meist in informellen und darüber hinaus emotional aufgeladenen Situationen/Momenten statt: , , [1]Language crossing was located in moments when the ordered flow of social life was loosened and normal social relations could not be taken for granted" (Rampton 1995:193, siehe hierzu auch Rampton 1995:281, 1997:65-69, 1998:298-299). Im Unterschied zum routinierten Code-Switching, das die Nebenexistenz von zwei Sprachen im alltäglichen Sprachgebrauch voraussetzt, ist Crossing aufgrund seiner Verwendung ,nur in begrenzten Momenten außerhalb der dominierenden sozialen Struktur“ nicht besonders „flexibel“ (vgl. Bücker 2007:15).

\subsection{Mehrstimmigkeit (Polyphonie) oder Double Voicing}

Neben dem Crossing-Phänomen kann auch Michail Bakhtins kultursemiotisches Konzept des Double Voicing (Bakhtin 1971) als eine prägnante Manifestation sprachlich-kultureller Hybridität angesehen werden. Der Terminus referiert auf die Koexistenz zweier Stimmen bzw. mehrerer Stimmen innerhalb einer Aussage, was sich in einer weiteren Bezeichnung für dasselbe Phänomen niederschlägt, und zwar in der Mehrstimmigkeit oder Polyphonie (vgl. Bakhtin 1971:202-228). Hierbei knüpft Bakhtins Wahrnehmung der Polyphonie an das Crossing-Konzept Ramptons. Genauer gesagt an eine seiner Ausprägungen, die als ironisches CodeSwitching definiert wird. Ausgehend von der Annahme, dass die Alltagssprache mit fremden Stimmen durchsetzt ist, prägt Bakhtin den Terminus der Mehrstimmigkeit (Polyphonie) als Integration von Versatzstücken sowie von ganzen Gesprächsphrasen aus einer anderen Sprache in die eigene Rede bzw. Übernahme von fremden, sprachlich codierten Werten, was für einen synkretistischen, sprich polyphonischen Charakter des Textganzen (der gesamten Aussage) sorgt (Bakhtin 1971:217). Eine Sonderform dieser Stimmenvielfalt stellt die unidirektionale Zweistimmigkeit dar, die als das gleichgerichtete zweistimmige Wort definiert wird und dem metaphorischen Code-Switching zugeordnet werden kann. Bei ihrer Umsetzung bedient sich der Sprecher einer fremden Stimme oder Sprache, um seine eigenen, veränderten Absichten oder Gefühlslagen vermitteln zu können. Die Übernahme von fremden sprachlichen Ressourcen lässt allmählich die Grenze zwischen Innen- und Außenbereich verschmelzen, worauf unmittelbar 
Ramptons Formel: die „Fusion der beiden Stimmen“ (Rampton 1998:304) oder aber auch Double Voicing hinweist. Während die unidirektionale Zweistimmigkeit ein gänzliches Ineinandergehen und eine vollkommene Durchdringung beider Stimmen in der Aussage eines Sprechers zulässt, bleiben bei der varidirektionalen Zweistimmigkeit (dem verschieden-gerichteten zweistimmigen Wort) Stimme und Sprecher eindeutig voneinander getrennt. Analog zum ironischen Code-Switching hält/pflegt man bei der varidirektionalen Zweistimmigkeit Distanz zu seinen Aussagen, indem die Stellungnahme zu bestimmten Sachverhalten spielerisch oder spöttisch abgegeben wird. In Anlehnung daran fügt Bücker Folgendes hinzu: „Aufgrund der eingeschränkten Gefahr, sich selbst zu blamieren oder andere zu beleidigen, verhilft diese Art der Polyphonie Sprechern dazu, sich vorbehaltloser und freier zu äußern“ (Bücker 2007:18, hervor. im Original).

\subsection{Transgression}

In Anlehnung an Rampton (1995) und Bakhtin (1971) definieren Ǐnci Dirm und Peter Auer Transgression als Überschreitung einer ethnisch-sozialen Grenze (vgl. Dirim/Auer 2004:27). Demnach deutet der Akt einer sprachbezogenen Grenzüberschreitung auf die Usurpierung einer Sprache bzw. einer Sprachvarietät, die den Grenzüberschreitern nicht gehört, hin. So gesehen, gilt Transgression als Act of Identity, die Wahl eines bestimmten Stils aus einem sozialen Stil-Repertoire andersstämmiger Gruppen (vgl. Dirim/Auer 2004:39). Hierbei kann das so gesehene Code-Switching (hier: Transgression) eine teilnehmer- oder diskursbezogene Funktion annehmen und heißt demgemäß teilnehmerbezogenes und diskursbezogenes Code-Switching (vgl. Dirim/Auer 2004:158-159).

Es sei an dieser Stelle betont, dass das Konzept von Dirim und Auer ein Sprachverhältnis zwischen türkisch- und deutschstämmigen Jugendlichen in der Bundesrepublik betrifft. In Anbetracht dessen könnte es interessant sein, zu fragen, warum die Grenzüberschreiter eine in der Migrantenszene entstandene Varietät der Majoritätssprache benutzen, der, wie es beim Türkenslang der Fall ist, ein geringes soziales Prestige ${ }^{8}$ innewohnt. Oder anders genannt, was sind die (Hinter-)Gründe für die damit ausgedrückten Acts of Identity? Gestützt auf historische Beispiele für Transgressionen dieser Art, also die Übernahme von Versatzstücken sowie ganzen Gesprächsphrasen aus den Sondersprecharten sozial benachteiligter Gruppen wie die Zigeuner (Romani), Landstreicher, fahrenden Händler, Viehhändler (Jenisch, Lekudesch) und Kleinbanditen (Rotwelsch) in die deutsche Studentensprache des 19. Jahrhunderts, kommen Dirim und Auer zur Erkenntnis, dass Transgressionen Distanz von der etablierten Mehrheitsgesellschaft mit ihren standradsprachlichen Normen markieren, die Identifizierung mit den primären

8 S. hierzu Wiese $(2012: 185,207)$. 
Trägern dieser Varietät jedoch grundsätzlich metaphorisch anzusehen ist (vgl. Dirim/Auer 2004:27). Denn die beiden Parteien kommen im Alltag nur wenig in Kontakt, nur selten handelt es sich bei Transgressionen um einen assimilatorischen (die gemeinsame Verständigung erleichternden) Spracherwebsprozess. Vielmehr wird mit der Übernahme und Integration von Neuzugängen aus anderen Sprachen und Sprachvarietäten in die eigene Rede die Selbstpositionierung im soziokulturellen Raum, Partizipation an einer großstädtischen Jugendkultur, die im Kontext der Bundesrepublik sprachlich und kulturell türkisch dominiert wirkt, gesichert (vgl. Dirim/Auer 2004:30, 146). Kurzum weisen Transgressionen nur bedingt auf die Solidarisierung mit gemischtethnischen und sozial benachteiligten Gruppen hin. Sie scheinen vielmehr auf deren sprachlich-kulturelle Produktivität ausgerichtet zu sein. Dass die Identifizierung mit einer bestimmten jugendkulturellen Szene die gleichzeitige Identifizierung mit deren Machern/Schöpfern herbeiführt, ist dabei aber nicht völlig auszuschließen.

So gesehen, sind Transgressionen Beispiele für Sonderformen des Ethnolekts, die dementsprechend als der sekundäre und der tertiäre Ethnolekt eingestuft werden. ${ }^{9}$ Während der sekundäre Ethnolekt die mediale Stilisierung des primären Ethnolekts darstellt, ,in die auch reine Comedy-Erfindungen wie der Universalartikel dem (dem ist korrekt) und neue Wortbildungen einflossen" (Riehl 2009:129, hervor. im Original), werden unter dem tertiären Ethnolekt Imitation und Verwendung des sekundären Ethnolekts von jugendlichen Sprechern nichttürkischer Herkunft verstanden. Die Jugendlichen anderer ethnischer Herkunft (darunter jene mit deutschem Familienhintergrund) greifen auf medial stilisierte Formen des primären Ethnolekts, ,um Dinge auszusprechen, die in der eigenen Stimme Gesichtsverletzung oder Tabubruch zum Ausdruck brächten“" (Riehl 2009:129). Allerdings kann der Türkenslang auch von denjenigen Jugendlichen nicht türkischer Herkunft benutzt werden, die enge Netzwerkbeziehungen zu Trägern des primären Ethnolekts haben. Ihr Gemischtsprechen ist Effekt von sprachlichen Kreuzungen und Mischungen, die tagtäglich in Migrantenvierteln der Bundesrepublik zum Vorschein kommen. Während beim sekundären und tertiären Ethnolekt die Überschreitung der sprachbezogenen Grenze (Transgression) metaphorisch verläuft und wie bereits zuvor angedeutet, nicht unbedingt auf die Affiliation mit den Türken ausgerichtet ist, handelt es sich beim Erwerb der Mischsprache ,aus erster Hand“ sowie deren Gebrauch keinerlei um doppeldeutige, sondern ganz umgekehrt - um routinierte, authentische und identitätsstiftende Manifestationen (Rituale).

9 Ausgehend von Jannis Androutsopoulos (2001b) entwirft Peter Auer die Typologie der ethnolektalen Formen des Deutschen (2003). In Bezug auf Sprechergruppen und die damit einhergehende Verwendung von ethnolektal geprägten Deutschvarietäten wird nach Auer zwischen dem primären, sekundären und tertiären Ethnolekt unterschieden. S. dazu noch Keim (2011:162). 


\section{Abschlussbemerkungen}

In der vorliegenden Arbeit wurde den wissenschaftlichen Arbeiten und Methoden zur Beschreibung des Gemischtsprechens Rechnung getragen. Hinzu wurden Motivationsgründe aufgezeigt, die gemischtethnische Gruppen zu Kreuzungen und Mischungen von sprachlich codierten Werten aus unterschiedlichen Kulturkreisen antreiben. Als Verwender der Mischsprache in der Bundesrepublik gelten vorwiegend Jugendliche deutscher und nicht deutscher (vor allem türkischer aber auch arabischer) Abstammung. Von ihnen gebrauchte Deutschvarietäten sind demnach ethnolektal, umgangs- und jugendsprachlich geprägt und fungieren als Ausdruck einer besonderen Solidarität mit sozial benachteiligten „Ghetto-Bewohnern“ ggf. der von ihnen gestalteten Jugendszene sowie falls stilisiert (montiert) und ironisch verfremdet völlig Distanzierung davon. Hierbei ist der sozial-symbolische Charakter der Imitation und Verwendung des sprachlich Gemischten für sämtliche Konzepte zur Beschreibung der Mehrsprachigkeit wie Bricolage, Hybridität, Language Crossing, Double Voicing und Transgression charakteristisch. Die besagten Konzepte wurden im vorliegenden Beitrag nicht nur in Bezug auf ihre Grundlagen, sondern auch ihre Etablierung in der bundesdeutschen Realität analysiert.

\section{Literaturverzeichnis}

ANDROUTSOPOULOS Jannis, 2001a, Ultra korregd Alder! Zur medialen Stilisierung und Aneignung von ,Türkendeutsch', in: Deutsche Sprache 29, S. $321-339$.

ANDROUTSOPOULOS Jannis, 2001b, From the streets to the screens and back again: On the mediated diffusion of ethnolectal patterns in contemporary German (= LAUD, Series A, 522), Essen.

ANDROUTSOPOULOS Jannis, 2003, jetzt speak something about italiano. Sprachliche Kreuzungen im Alltagsleben, in: Erfurt J. (Hrsg.), „Multisprech“: Hybridität, Variation, Identität. Oldenburg, S. $79-109$.

AUER Peter, 2003, „Türkenslang“ - ein jugendsprachlicher Ethnolekt des Deutschen und seine Transformationen, in: Häcki-Buhofer A. (Hrsg.), Spracherwerb und Lebensalter. Tübingen/ Basel, S. 255-264.

BAKHTIN Michail M., 1971, Probleme der Poetik Dostoevskijs, München.

BECKER Angelika / KLEIN Wolfgang, 1984, Notes on the internal organisation of a learner variety, in: Auer P./di Luzio A. (Hrsg.), Interpretive Soziolonguistics, Tübingen, S. 215 -232.

BLOM Jan-Petter / GUMPERZ John J., 1972, Social meaning in linguistic structure: code-switching in Norway, in: Gumperz J.J./Hymes D. (eds.), Directions in Sociolinguistics. The Ethnography of Communication, New York, S. $407-434$.

BodEMANN Y. Michal / OstRow Robin, 1975, Lingua franca und Pseudo-Pidgin in der Bundesrepublik: Fremdarbeiter und Einheimische im Sprachzusammenhang, in: LiLi 5 (17-19/20), S. 122-146.

BOURDIEU Pierre, 1982 (franz. 1979), Die feinen Unterschiede. Kritik der gesellschaftlichen Urteilskraft, Frankfurt am Main.

BÜCKER Tanja, 2007, Ethnolektale Varietäten des Deutschen im Sprachgebrauch Jugendlicher, SASI Heft 9, Münster. 
CANOĞLU Hatice Deniz, 2012, Kanak Sprak versus Kiezdeutsch - Sprachverfall oder sprachlicher Spezialfall? Eine ethnolinguistische Untersuchung, Berlin.

ClARKE John, 1981, Stil, in: Clarke J./Honneth A. (Hrsg.), Jugendkultur als Widerstand, 2. Aufl., Frankfurt am Main, S. $133-157$.

CLYNE Michael, 1968, Zum Pidgin-Deutsch der Gastarbeiter, in: Zeitschrift für Mundartforschung 35, S. $130-139$.

DIRIM Ǐnci / AUER Peter, 2003, „Mit der Zeit versteht man alles“ - Zum ungesteuerten Erwerb des Türkischen durch Jugendliche nicht-türkischer Herkunft, in: Erfurt J. (Hrsg.), „Multisprech“: Hybridität, Variation, Identität, Oldenburg, S. 57-78.

DIRIM Ǐnci / AUER Peter, 2004, Türkisch sprechen nicht nur die Türken: Über die Unschärfebeziehung zwischen Sprache und Ethnie in Deutschland, Berlin.

DITTMAR Norbert, 1982, „Ich fertig arbeite - nicht mehr spreche Deutsch“. Semantische Eigenschaften pidginisierter Lernervarietäten des Deutschen, in: LiLi 45, S. 84-103.

ERFURT Jürgen, 2003, „Multisprech“: Hybridität, Variation, Identität, Oldenburg.

EsteVA-FABREGat Claudio, 1995 [1987], Mestizaje in Ibero-America. Tucson/London [span. Original: Mestizaje en Iberoamerica, 1987].

GARCIÁ Canclini Nestor, 1995 [1987], Hybrid Cultures. Strategies of Entering and Leaving Modernity. Minneapolis/London [span. Original: Culturas hibridas: Estrategias para entrar y salir de la modernidad, 1989].

GoGOLIN Ingrid, 1994, Der monolinguale Habitus der multilingualen Schule, Münster/New York.

GrIEM Julika, 2008, [Art.] Hybridität, in: Ansgar N. (Hrsg.), Metzler Lexikon Literatur- und Kulturtheorie. 4., aktualisierte und erweiterte Auflage, Stuttgart/Weimar, S. 297.

GUMPERZ John J., 1982, Discourse strategies, Cambridge.

GUMPERZ John J., 1994, Ethnography in urban communication, in: Auer P./di Luzio A. (Hrsg.), Interpretive sociolinguistics. Migrants-children-migrant children, Tübingen, S. 1-12.

HEIDELBERGER Forschungsprojekt „Pidgin-Deutsch“, 1975, Sprache und Kommunikation ausländischer Arbeiter, Kronberg/Taunus.

HEWITT Roger, 1986, White talk black talk. Inter-racial friendship and communication amongst adolescents, Cambridge.

HewITT Roger, 1998, Ethnizität in der Jugendkultur, in: Gogolin I. et al. (Hrsg.), Pluralität und Bildung, Opladen, S. $13-24$.

HiNNENKAMP Volker, 1982, Foreigner Talk und Tarzanisch. Eine vergleichende Studie über die Sprechweise gegenüber Ausländern am Beispiel des Deutschen und des Türkischen, Hamburg.

HINNENKAMP Volker, 1998, Mehrsprachigkeit in Deutschland und deutsche Mehrsprachigkeit. Szenarien einer migrationsbedingten Nischenkultur der Mehrsprachigkeit, in: Kämper H./Schmidt H. (Hrsg.), Das 20. Jahrhundert: Sprachgeschichte - Zeitgeschichte, Berlin, S. 137-162.

HINNENKAMP Volker, 2000, „Gemischt sprechen“ von Migrantenjugendlichen als Ausdruck ihrer Identität, in: Der Deutschunterricht 5, S. 96-107.

HINNENKAMP Volker, 2005, Einleitung, in: Hinnenkamp V./Meng K. (Hrsg.), Sprachgrenzen überspringen. Sprachliche Hybridität und polykulturelles Selbstverständnis, Tübingen, S. 7-18.

HINNENKAMP Volker, 2007, Vom Nutzen einer hybriden Sprache. in: Bukow W.-D./Nikodem C./ Schulze E./Yildiz E. (Hrsg.), Was heißt hier Parallelgesellschaft? Zum Umgang mit Differenzen, Wiesbaden, S. 175-199.

KALlMEYER Werner, 1994, Kommunikation in der Stadt. Teil 1: Exemplarische Analysen des Sprachverhaltens in Mannheim (= Schriften des Instituts für deutsche Sprache 4.1), Berlin.

KALLMEYER Werner, 1995, Kommunikation in der Stadt. Teil 2: Ethnographien von Mannheimer Stadtteilen (= Schriften des Instituts für deutsche Sprache 4.2), Berlin.

KALLMEYER Werner / KEIM Inken, 2003, Eigenschaften von sozialen Stilen der Kommunikation: Am Beispiel einer türkischen Migrantinnengruppe, in: Erfurt J. (Hrsg.), „Multisprech“: Hybridität, Variation, Identität, Oldenburg, S. $35-56$. 
KEIM Inken, 1978, Gastarbeiterdeutsch. Untersuchungen zum sprachlichen Verhalten türkischer Gastarbeiter, Tübingen.

KeIM Inken, 1995, Kommunikation in der Stadt. Teil 3: Kommunikative Stilistik einer sozialen Welt »kleiner Leute« in der Mannheimer Innenstadt (= Schriften des Instituts für deutsche Sprache 4.3), Berlin.

KEIM Inken, 2004, Die Verwendung medialer Stilisierungen von Kanaksprak durch Migrantenjugendliche, in: Kodikas/Code. Ars: Semiotica, Vol. 26 (2003)/1 - 2, S. $97-111$.

KEIM Inken, 2008, Die „türkischen Powergirls“. Lebenswelt und kommunikativer Stil einer Migrantinnengruppe in Mannheim, Tübingen.

KEIM Inken, 2011, Form und Funktion ethnolektaler Formen: türkischstämmige Jugendliche im Gespräch, in: Eichinger L.M./Plewnia A./Steinle M. (Hrsg.), Sprache und Integration. Über Mehrsprachigkeit und Migration, Tübingen, S. 157-188.

KEIM Inken, 2012, Mehrsprachige Lebenswelten. Sprechen und Schreiben der türkischstämmigen Kinder und Jugendlichen, Tübingen.

KeIM Inken / NiKITOPOULOS Pantelis / REPP Michael, 1982, Kommunikation ausländischer Arbeiter. Eine Studie zum deutschsprachigen Interaktionsverhalten von griechischen und türkischen Arbeitern, Tübingen.

KERN Friederike / SELTING Margret, 2006, Einheitenkonstruktion im Türkendeutschen: Grammatische und prosodische Aspekte, in: Zeitschrift für Sprachwissenschaft 25, S. 239-272.

KERN Friederike / ŞIMŞEK Yazgül, 2006, Türkendeutsch: Aspekte von Einheitenbildung und Rezeptionsverhalten, in: Wolff D. (Hrsg.), Mehrsprachige Individuen - vielsprachige Gesellschaften, Frankfurt am Main, S. $101-119$.

LESCH-SCHUMACHER Barbara / SCHUMACHER Michael, 2009, »Kiezsprache, Türkenslang, Kanak Sprak? Ethnolekt im Gegenwartsdeutsch «, in: RAAbits Deutsch/Sprache 61.

MARKERT Svenja, 2009, Kommunikative Nähe und Stilisierung von Jugendlichkeit, in: Arbeitspapiere und Materialien zur deutschen Sprache, Band 36, Mannheim.

MEISEL Jürgen, 1975, Ausländerdeutsch und Deutsch ausländischer Arbeiter. Zur möglichen Entstehung eines Pidgin, in: Zeitschrift für Literaturwissenschaft und Linguistik (LiLi), Heft 18, S. $9-53$.

NeLDE Peter, 1998, Migrantensprachen, in: Holtus G./Metzeltin M./Schmitt Ch. (Hrsg.), Lexikon der romanistischen Linguistik, Bd. VII, Tübingen, S. 518-526.

RAMPTON Ben, 1995, Crossing. Language and ethnicity among adolescents, London.

RAMPTON Ben, 1997, Sociolinguistics and Cultural Studies: New Ethnicities, Liminality and Interaction, in: Social Semiotics 1999/9(3), S. 355-374.

RAMPTON Ben, 1998, Language crossing and the redefinition of reality, in: Auer P. (Hrsg.), CodeSwitching in Conversation, London, S. 290-320.

RIEHL Claudia Maria, 2009, Sprachkontaktforschung: Eine Einführung, Tübingen.

Rincón Carlos, 1994, Die neuen Kulturtheorien, in: Scharlau B. (Hrsg.), Lateinamerika denken. Kulturtheoretische Grenzgänge zwischen Moderne und Postmoderne, Tübingen, S. 1-35.

RochE Jörg, 1989, Xenolekte: Struktur und Variation im Deutsch gegenüber Ausländern, Berlin.

SCHLOBINSKI Peter / HeINS Niels-Christian (Hrsg.), 1998, Jugendliche und ,ihre“ Sprache: Sprachregister, Jugendkulturen und Wertesysteme, Opladen/Wiesbaden.

SCHLOBINSKI Peter / KOHL Gaby / LUDEWIGT Irmgard, 1993, Jugendsprache: Fiktion und Wirklichkeit, Opladen/Wiesbaden.

SCHÜTZE Fritz, 1987, Symbolischer Interaktionismus, in: Ammon U. et al. (Hrsg.), Sociolinguistics. An International Handbook of the Science of Language and Society $(3,1$. Halbband), Berlin/ New York, S. 520 - 553.

Schwitalla Johannes, 1988, Die vielen Sprachen der Jugendlichen, in: Gutenberg N. (Hrsg.) Kann man Kommunikation lehren? (= Sprache und Sprechen, Bd. 19), Frankfurt am Main, S. $167-176$. 
SCHWitalla Johannes, 1994, Poetisches in der Alltagskommunikation, in: Halwachs D. et al. (Hrsg.), Sprache, Onomatopöie, Rhetorik, Namen, Idiomatik, Grammatik, Graz, S. 227-243.

SchwitALla Johannes, 1995, Kommunikation in der Stadt. Teil 4: Kommunikative Stilistik zweier sozialer Welten in Mannheim-Vogelstang (= Schriften des Instituts für deutsche Sprache 4.4), Berlin.

ŞIMŞEK Yazgül, 2012, Türkendeutsch: Formen und strukturelle Merkmale des Sprachgebrauchs türkisch-deutscher Jugendlicher aus Berlin, in: Jańczak B./Jungbluth K./Weydt H. (Hrsg.), Mehrsprachigkeit aus deutscher Perspektive, Tübingen, S. 155-180.

SPECHT Theresa, 2011, Transkultureller Humor in der türkisch-deutschen Literatur, Würzburg.

STRAUSS Anselm L., 1968, Spiegel und Masken. Die Suche nach Identität, Frankfurt am Main.

WIESE Heike, 2006, „Ich mach dich Messer“. Grammatische Produktivität in Kiez-Sprache, in: http:// publikationen.ub.uni-frankfurt.de/frontdoor/index/index/docId/12791, (Zugriff am 12.05.2015).

WIESE Heike, 2009, Grammatical innovation in multiethnic urban Europe: New linguistic practises among adolescents, in: Lingua 119, S. 782-806.

WIESE Heike, 2012, Kiezdeutsch. Ein neuer Dialekt entsteht, München.

YAKUT Atilla, 1981, Sprache der Familie. Eine Untersuchung des Zweitsprachenerwerbs der türkischen Gastarbeiterfamilien in der BRD, Tübingen.

ZAIMOĞLU Feridun, 1995, Kanak Sprak. 24 Mißtöne vom Rande der Gesellschaft, Hamburg.

ZAIMOĞLU Feridun, 1997, Abschaum. Die wahre Geschichte von Ertan Ongun, Hamburg.

ZAPF Harald, 2002, Dekonstruktion des Reinen. Hybridität und ihre Manifestationen im Werk von Ishmael Reed, Würzburg. 